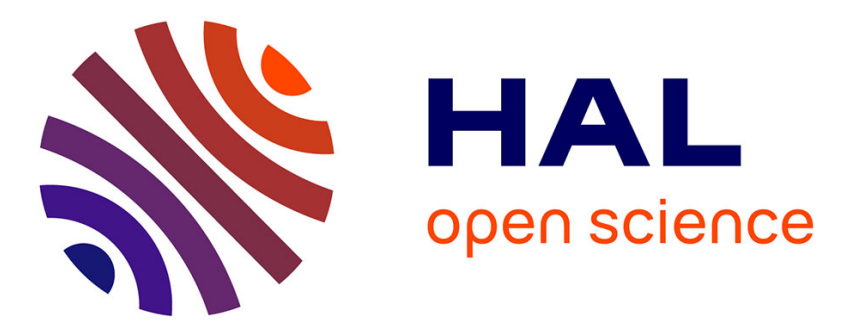

\title{
A pulsed high-voltage decelerator system to deliver low-energy antiprotons
}

\author{
A. Husson, B.H. Kim, A. Welker, M. Charlton, J.J. Choi, M. Chung, P. \\ Cladé, P. Comini, P.-P. Crépin, P. Crivelli, et al.
}

\section{- To cite this version:}

A. Husson, B.H. Kim, A. Welker, M. Charlton, J.J. Choi, et al.. A pulsed high-voltage decelerator system to deliver low-energy antiprotons. Nucl.Instrum.Meth.A, 2021, 1002, pp.165245. 10.1016/j.nima.2021.165245 . hal-03203652

\section{HAL Id: hal-03203652 \\ https://hal.science/hal-03203652}

Submitted on 22 Jul 2021

HAL is a multi-disciplinary open access archive for the deposit and dissemination of scientific research documents, whether they are published or not. The documents may come from teaching and research institutions in France or abroad, or from public or private research centers.
L'archive ouverte pluridisciplinaire HAL, est destinée au dépôt et à la diffusion de documents scientifiques de niveau recherche, publiés ou non, émanant des établissements d'enseignement et de recherche français ou étrangers, des laboratoires publics ou privés. 


\title{
A pulsed high-voltage decelerator system to deliver low-energy antiprotons
}

The GBAR Collaboration

(author list appended)

\begin{abstract}
The GBAR (Gravitational Behaviour of Antihydrogen at Rest) experiment at CERN requires efficient deceleration of $100 \mathrm{keV}$ antiprotons provided by the new ELENA synchrotron ring to synthesize antihydrogen. This is accomplished using electrostatic deceleration optics and a drift tube that is designed to switch from $-99 \mathrm{kV}$ to ground when the antiproton bunch is inside - essentially a charged-particle "elevator" - producing a $1 \mathrm{keV}$ pulse. We describe the simulation, design, construction and successful testing of the decelerator device at $-92 \mathrm{kV}$ on-line with ELENA.
\end{abstract}

Keywords: Antihydrogen, General Relativity, Charged-particle optics, ion-optic simulations

\section{Introduction}

The GBAR (Gravitational Behaviour of Antihydrogen at Rest) experiment [1] at CERN aims at testing the Weak Equivalence Principle (WEP) of General Relativity by measuring the free fall of antihydrogen $(\overline{\mathrm{H}})$ in the Earth's gravitational field. The WEP has been stringently tested in different regimes but never using antimatter, which may fall with a different acceleration than for matter. Such an experiment requires extremely well-defined initial conditions, with near-zero initial velocity. While this goal is likewise pursued by the CERN experiments ALPHA [2] and AEgIS [3], GBAR will attempt a unique approach by synthesizing antihydrogen ions $\left(\overline{\mathrm{H}}^{+}\right)$that can be sympathetically cooled by coupling to a laser-cooled trapped-Be-ion crystal $[4,5,6]$, reaching velocities of about $1 \mathrm{~m} / \mathrm{s}(60 \mu \mathrm{K})$.

GBAR will fabricate antihydrogen using the electron-positron atomic system, positronium (Ps) created by directing positrons onto a mesoporous sil- 
ica target [7]. Antiprotons sent through the Ps cloud undergo two successive charge-exchange reactions, forming $\overline{\mathrm{H}}$ and $\overline{\mathrm{H}}^{+}$. Producing $\overline{\mathrm{H}}^{+}$requires a higher positron flux than achievable with a radioactive source. Therefore, the positrons required to form a Ps cloud of sufficient density are generated using a $9 \mathrm{MeV}$ electron linear accelerator [8], cooled in a buffer-gas magnetic trap and then accumulated in a $5 \mathrm{~T}$ Penning-Malmberg trap [9] before being directed onto the target.

The Ps reaction for the formation of antihydrogen was proposed by Humberston et al. [10] and first cross-section measurements were performed by Merrison et al. [11] at energies down to $11.3 \mathrm{keV}$. The predictions of various atomic-physics models reported in [11] did not agree on the most favorable energy but more recent calculations $[12,13]$ predict that even lower antiproton energies would lead to higher cross sections, hence better $\overline{\mathrm{H}}$ and $\overline{\mathrm{H}}^{+}$ production rates. Refined calculations are in progress to probe this important question [14].

The $\overline{\mathrm{H}}^{+}$ions for GBAR will be fabricated using antiprotons delivered by the CERN AD-ELENA facility [15]. The ELENA synchrotron heralds a new era of antiproton and antimatter physics. Its deceleration of antiprotons from 5.3 MeV to $100 \mathrm{keV}$ bridges an important gap to achieve more efficient antihydrogen fabrication and storage at sub-Kelvin temperatures.

The first capture of antiprotons into a Penning trap was performed by Gabrielse et al. [16] using a beryllium energy-degrader foil for a $150 \mathrm{~ns}$ burst of $10^{8}$ antiprotons delivered at $21.3 \mathrm{MeV}$ by CERN's LEAR facility. CERN's AD facility later provided pulses of $3 \times 10^{7}$ antiprotons at 5.3 $\mathrm{MeV}$, allowing the use of thinner foils to achieve rates of about 20000/shot [17]. The ASACUSA experiment further reduced this energy to below 100 $\mathrm{keV}$, using a radiofrequency quadrupole linear accelerator operated in reverse mode $[18,19,20]$. The lower incident beam energy allowed the use of foils that were 800 times thinner than in [16] and improved the number of trapped antiprotons by a factor of 50 [19].

Using electrostatic deceleration would ideally avoid all losses associated with the use of foils. ELENA now allows using electrostatic retardation and fast switching to reach the energy regime favorable for antihydrogen formation by charge exchange.

This article describes a novel multi-electrode deceleration system that creates a $100 \mathrm{keV}$ particle "elevator". This is accomplished using electrostatic retardation optics and a drift tube that is switched from $-99 \mathrm{kV}$ to ground during the short time that the antiproton bunch is inside. Further optics 
focus the low-energy beam into the Ps reaction chamber. Simulations for the optimization of the potential values are described, as well as the design, construction and preliminary testing of the decelerator device down to $-92 \mathrm{kV}$ using $\mathrm{H}^{-}$and antiprotons from ELENA during its commissioning period.

\section{Design and construction of the decelerator}

The concept of the decelerator and results from first tests with the prototype have been described in $[21,22,23]$. The principle is to use a static electric field to slow the charged antiprotons into a drift tube, within which there are ideally no potential gradients. Once the antiproton pulse is inside the field-free region, the voltage applied to the tube is switched to ground. If the switching is fast enough, there will be no voltage gradient at the exit of the tube when the antiprotons arrive, so they continue at their decelerated kinetic energy. Of course the use of drift tubes for changing the energy of particle beams is not new. Drift tubes form the heart of linear accelerators using AC voltages, however their use as particle elevators is less common. The so-called pulsed drift tube is used at many nuclear physics facilities where ion species are transported with energies of 30-60 keV and must be slowed to a few eV to be confined in a trap. The deceleration of the ion bunch causes the beam emittance to blow up so that the ions must be cooled to be trapped. The technique was largely developed by the ISOLTRAP experiment at CERN's ISOLDE facility, in conjunction with a device for accumulating ISOLDE beams [24]. In this scheme, the beam is decelerated into a linear radiofrequency trap filled with buffer gas, which cools the large emittance of the decelerated beam to essentially a point-like source. The drift tube is mounted directly downstream of the trap [25]. This scheme is now used by several on-line trap facilities.

The main difference with the decelerator presented here is that since antiprotons would annihilate with the buffer gas atoms the deceleration process must include optics to keep the larger emittance within the drift-tube electrode and limit divergence in the region of potential gradients.

A deceleration system for ion beams that did not incorporate a gas-filled cooler buncher was designed for the WITCH experiment at ISOLDE by Coeck et al. [26]. The beam was cooled in a gas-filled Penning trap a few meters upstream and reaccelerated. The drift tube was almost $700 \mathrm{~mm}$ long and used an intermediate deceleration electrode to limit the beam divergence, decelerating $43 \%$ of the incident $30 \mathrm{keV}$ beam with $24 \mathrm{kV}$ on the drift 
tube. A similar scheme for $30 \mathrm{keV}$ ions was developed for the TRIGATRAP experiment in Mainz [27].

Decelerating $100 \mathrm{keV}$ ELENA beams requires a substantial scaling from the $30 \mathrm{kV}$ systems mentioned above.

ELENA was designed to provide antiproton bunches of $300 \mathrm{~ns}$ duration (defined as four times rms value of 75 ns that includes $95 \%$ of an approximately Gaussian distribution), $0.25 \%$ momentum spread and about $4 \pi \mathrm{mm}$ mrad transverse emittance [15]. When the $100 \mathrm{keV}$ beam is decelerated to $1 \mathrm{keV}$, the transverse emittance increases to $40 \pi \mathrm{mm} \mathrm{mrad}$ and the $1.3 \mathrm{~m}$ pulse length is reduced to about $150 \mathrm{~mm}$. We consequently chose a drift-tube length of $400 \mathrm{~mm}$ to allow enough time for switching within a field-free region.

The first consequence of decelerating the beam is a large divergence, which will cause huge losses inside a long drift tube. It is also critical to preserve good focusing properties of the beam by avoiding aberrations during the deceleration. This requires keeping the beam as parallel as possible, especially where the field gradient is large, and necessitates extra degrees of freedom for the optics. The work of C. Smorra for the TRIGATRAP drift tube [28] gives an excellent illustration.

Simulations were performed using the ion-optics program SIMION ${ }^{\circledR}$, starting with three electrodes (in the familiar einzel lens geometry). The decelerated beam developed a rather large diameter, introducing aberrations that prevented good focusing (the GBAR experiment requires directing the decelerated beam into a $1 \mathrm{~mm}$ diameter cavity). This solution also led to large variations in divergence for small changes in deceleration voltage. Therefore a second triplet geometry was introduced to provide more flexibility keeping the diameter smaller. We also reasoned that any of the additional lenses could always be grounded if they were not necessary. It is interesting to note that the work of Coeck et al. [26] concluded with the proposition of including extra electrodes to improve their decelerator design.

An example of a SIMION ${ }^{\circledR}$ trajectory calculation is shown in Fig. 1, which refocuses the decelerated beam near the entrance of the drift tube. The antiproton beam arrives from the left at $100 \mathrm{keV}$ and exits the drift tube with only $1 \mathrm{keV}$ since the voltage on the tube is switched to ground while the ion bunch is inside. Despite different combinations of the different voltages, the output beam is relatively divergent so that an additional Einzel lens is required downstream.

In addition to steering electrodes, the ELENA LNE50 extraction beamline 
includes two quadrupole doublets, which can produce a convergent, parallel, or diverging beam at the decelerator entrance. The trajectories shown in Fig. 1 were calculated with the parallel ELENA beam, which was found to be the most favorable. The beam diameter is $18 \mathrm{~mm}$.

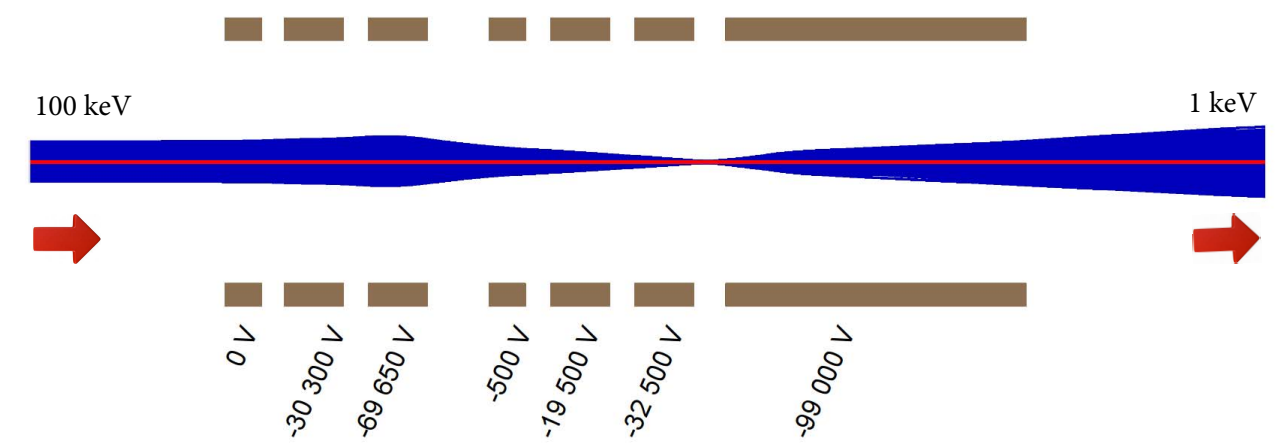

Figure 1: Antiproton trajectories in the decelerator (travelling left to right), calculated by the SIMION ${ }^{\circledR}$ program for the potentials indicated on the six (100 $\mathrm{mm}$ diameter) electrodes that slow and focus the beam. The potential on the drift tube $(-99 \mathrm{kV})$ is switched to ground when the antiproton pulse is inside. Input beam parameters are the nominal ELENA values given in the text. The output beam energy is $1 \mathrm{keV}$.

A drawing of the decelerator system vacuum chambers and electrodes is shown in Fig. 2. The dimensions were chosen so that the electrodes are large enough (100 mm diameter) to accept the beam without difficulty but far enough from the DN250CF chamber walls to avoid sparking. The deceleration electrodes are arranged as triplets, insulated via MACOR ${ }^{\circledR}$ (Machinable glass ceramic by Corning) to a support frame in the chamber. The overall length is $1225 \mathrm{~mm}$ with an additional $225 \mathrm{~mm}$ chamber housing a low-energy Einzel-lens assembly to focus the decelerated beam into the GBAR reaction chamber downstream.

From the simulations shown in Fig.1 relatively high voltages are required on the decelerator electrodes, making the geometry and connections nontrivial. It is critical not to place wires near the chamber walls and to avoid any sharp edges. Photographs of the second electrode set and the pulsed drift tube are shown in Fig. 3. The drift tube, required to hold $-100 \mathrm{kV}$, is supported by a specially designed "cradle" (Fig. 3, right) made from MACOR ${ }^{\circledR}$ to avoid the presence of any metallic surfaces. The connection was made using a metal rod, screwed perpendicularly into the drift tube itself. 


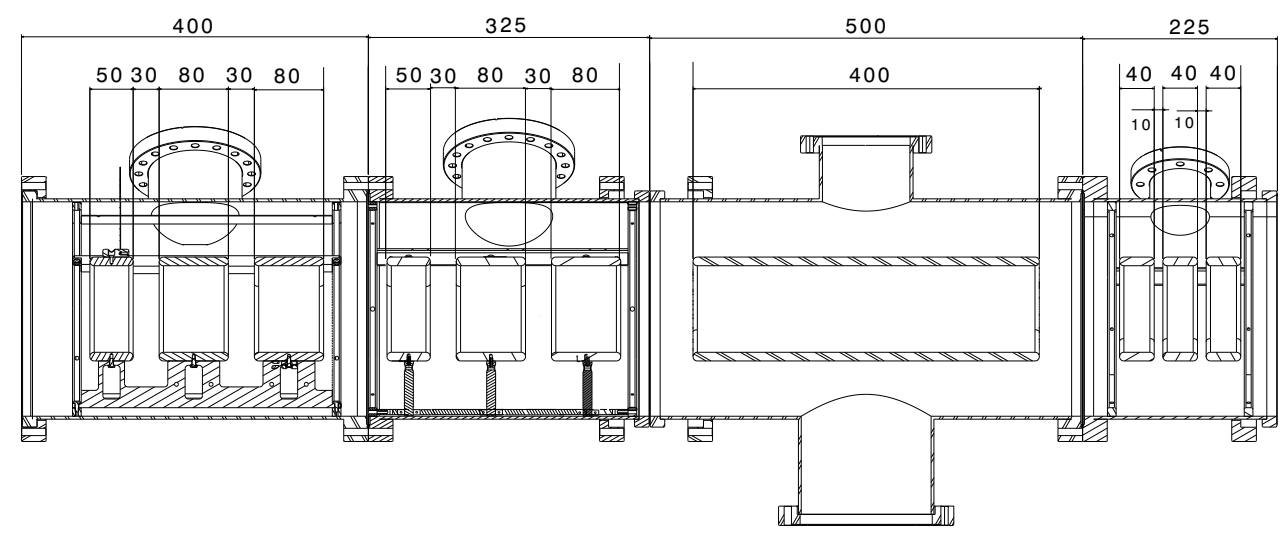

Figure 2: Computer-aided design drawing of the DN250CF vacuum chambers with electrode lengths indicated (all diameters are $100 \mathrm{~mm}$ ).

Connecting the power supplies to the high-voltage vacuum feedthroughs required embedding the wire inside a rounded bushing, since the threads on the feedthroughs create corona discharge in air if not covered. Because of the exceptionally high voltage on the drift tube, the air-side feedthrough was connected inside a hollow (brass) metal ball. Connecting the drift tube to the high-voltage switch (150 kV Behlke model HTS 1501-20-LC2) was done through high-voltage resistors, each of which was connected using metallic balls to avoid edges. A photograph of the switching circuit is shown in Fig. 4 along with the schematic diagram. The various elements are clad with teflon for increased protection against discharges (see inset of Fig. 4) and enclosed in a copper box for electromagnetic shielding.

Three high-voltage, non-inductive (Nicrom Electronics, series 500) resistors are visible in Fig. 4. A 1 G $\Omega$ resistor (right) avoids short circuiting the power supply while switching and limits the charging current while slowing the charging time to avoid sparking. After discharge damage that prevented applying the full voltage, this resistor was changed to $200 \mathrm{M} \Omega$ to compensate for the leakage. The vertical $1 \mathrm{k} \Omega$ resistor limits transient currents to the admissible rating of the switch (150 A). Finally, a $120 \Omega$ resistor (left) matches the switch impedance and stray capacitance to the load. We obtained a risetime of roughly $200 \mathrm{~ns}$.

Each electrode lens of the decelerator is connected to a separate power supply, which is controlled via a LabVIEW ${ }^{\circledR}$ program and a National Instruments compact-DAQ interface. An essential step before using the de- 


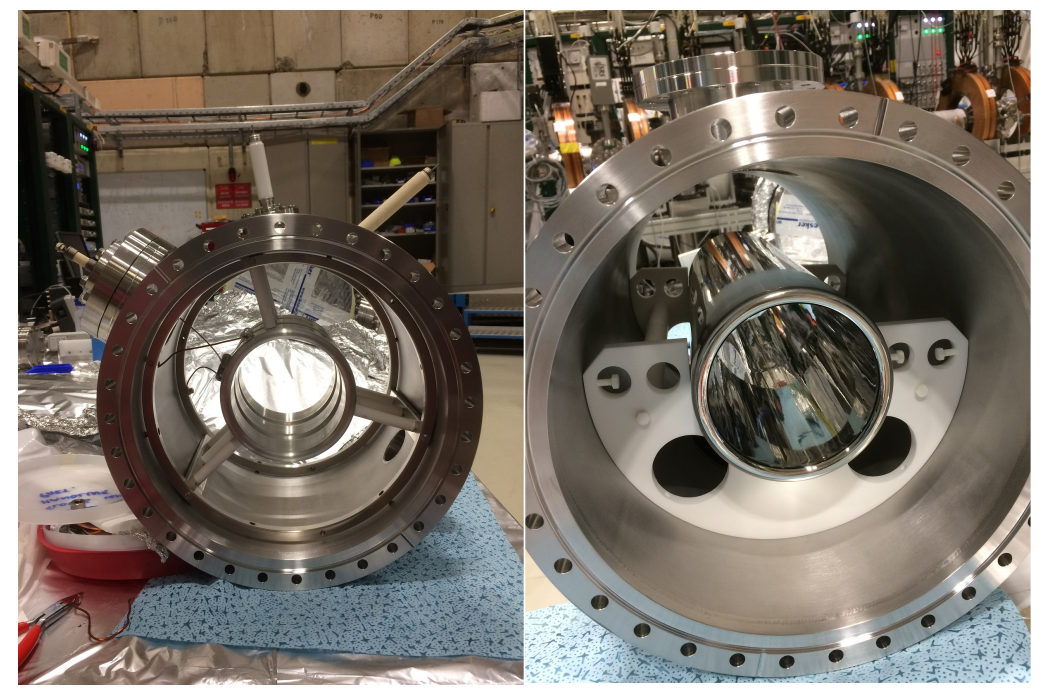

Figure 3: Photographs of: (left) the second set of decelerating electrodes, mounted in their $250 \mathrm{CF}$ vacuum chamber on MACOR ${ }^{\circledR}$ stand-offs; (right) the pulsed drift tube, machined from stainless steel, mounted on a MACOR ${ }^{\circledR}$ "cradle" for stability and insulation.

celerator is the high-voltage conditioning of each electrode. When applying over $10 \mathrm{kV}$ to an electrode, contamination on the surfaces provokes small discharges which cause spikes in vacuum pressure. The high-voltage conditioning actually cleans and helps outgas the surfaces but must not be rushed since an arc easily leaves a trace that can be impossible to burn away. After mounting and baking the chambers, this process took several hours for each electrode to reach its required voltage.

A photograph of the decelerator system vacuum chambers connected to the ELENA beamline is shown in Fig. 5. A $300 \mathrm{l} / \mathrm{s}$ ion pump is mounted under the first set of deceleration electrodes and a vacuum of $1 \times 10^{-9} \mathrm{mbar}$ was achieved after baking to about 200 degrees Celsius. (Note that neither pump nor port are shown in Fig. 2.)

\section{Commissioning tests}

The decelerator system was first tested off-line with a $50 \mathrm{keV}$ proton beam, provided by a Penning discharge source and hydrogen-gas leak. A description of the test bench with results from time-of-flight and energydispersion tests were reported in $[22,23]$. First tests at CERN were carried 




Figure 4: Photograph of the switching circuit, high-voltage connections, resistors (grey) and switch (black). Connection to the $100 \mathrm{kV}$ supply is on the right. Inset photograph shows the resistors with their teflon cladding, installed in a copper Faraday cage. Lower insert shows the circuit diagram (values discussed in the text).

out using a $\mathrm{H}^{-}$plasma source connected to ELENA. The $\mathrm{H}^{-}$beam was injected into the ring at $85 \mathrm{keV}$ (sparking in the isolation transformer prevented higher beam energy). After several turns in the ring, the beam was kicked into the LNE50 extraction beamline to the decelerator, with its electrodes grounded. A combination of possible beam optics and diagnostic alignment problems seems to have prevented the LNE50 quadrupoles from focusing the beam through the first apertures of the decelerator line. As a result, we were not able to transport the beam to the decelerator with the calculated nominal optical element values.

We also performed tests with antiproton bunches decelerated from 5.3 $\mathrm{MeV}$ by the ELENA storage ring and delivered at $100 \mathrm{keV}$. While the AD cycle for antiprotons is much slower (one pulse every $110 \mathrm{~s}$, compared to about every $5 \mathrm{~s}$ using the $\mathrm{H}^{-}$source) the antiproton annihilation detected by scintillators along the beamline provides an excellent diagnostic for beam 


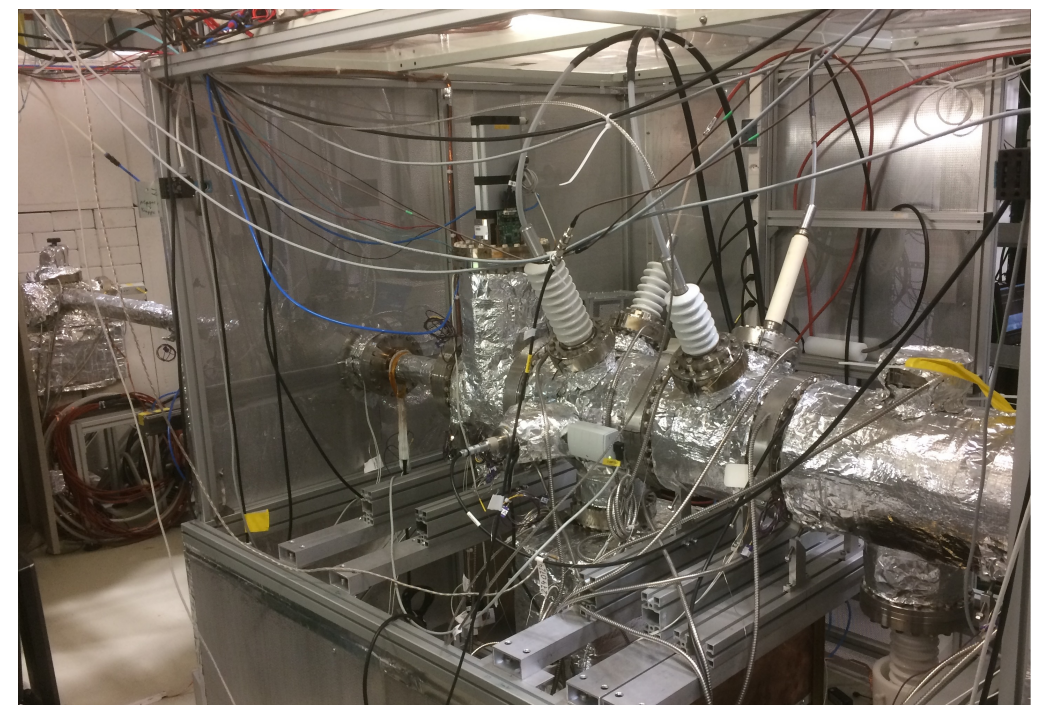

Figure 5: Photograph of the installed decelerator system and high-voltage connections (see technical drawing in Fig. 2). The $100 \mathrm{kV}$ Behlke switch is located below the system. The beamline connected to the ELENA extraction line (LNE50) is visible on the left side, as is the white wall separating the GBAR and ELENA zones. The high-voltage protection cage is also visible (the foreground panel was removed for the photograph).

losses. The deceleration of ELENA antiprotons is illustrated by the following discussion and figures. First, Fig. 6 illustrates the GBAR beam-line elements, detectors and relative distances after the handover point at the end of the LNE50 beamline (the wall can be seen in the photograph of Fig. 5). The po-

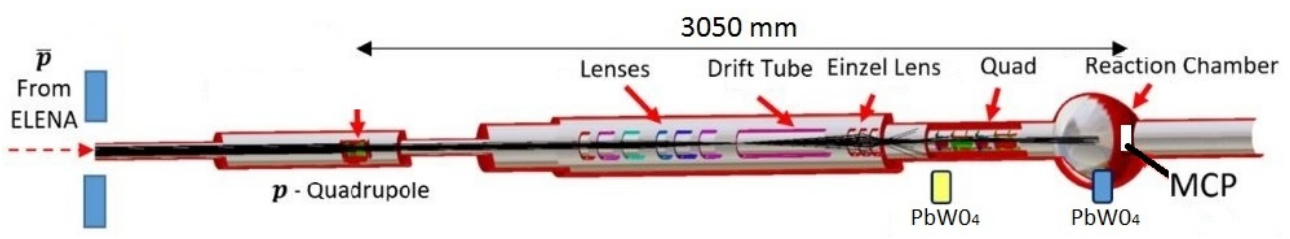

Figure 6: Schematic view of the decelerator optical elements, $\mathrm{PbW0}_{4}$ scintillator detectors and the MCP detector (lenses and drift tube are as in Figure 1). The distance from the end of the drift tube to the MCP is $1170 \mathrm{~mm}$ and from the upstream scintillator to the MCP is $700 \mathrm{~mm}$.

sition marked "p-Quadrupole" in Fig. 6 is a $12 \mathrm{~mm}$ horizontal collimation of an electrostatic quadrupole bender used to steer protons in from a 90-degree angle. During beam tuning with the MCP imaging detector, the shadow of 
this collimator was clearly visible. With antiprotons an additional scintillator (not shown in the figure) recorded a relatively large annihilation signal, the time of which corresponded to the time of flight of the $100 \mathrm{keV}$ antiprotons from the ELENA deflector. We estimated the resulting transmission to be about $25 \%$.

As the tests were performed in parallel with the commissioning of the ELENA machine, it was not possible to perform systematic studies. A critical parameter for successful deceleration of the beam pulse is the timing of the drift-tube switch with respect to the ELENA extraction (an electrostatic septum that is switched from ground to a given extraction voltage). This pulse was fed into a digital delay generator to generate the trigger for the switch.

Fig. 7 shows screen shots of the scintillator and MCP detector oscilloscope traces and MCP antiproton-beam images (inset). The top panel was recorded with the trigger too early and the bottom panel with the trigger too late. An early trigger means that the drift tube is pulsed to ground before the antiproton pulse arrives. Therefore the antiprotons see no potential on the drift tube and experience no deceleration. The late trigger causes the drift tube voltage to remain at its set value (here it was $-90 \mathrm{kV}$ ) during the transit of the antiproton pulse. This results in an initial deceleration of the pulse and reacceleration to $90 \mathrm{keV}$ after transiting the drift tube at $10 \mathrm{keV}$. (There were no voltages on the other electrodes for this measurement.) The difference in time of flight between the full-energy beam and slowed/re-accelerated beam is calculated to be about $200 \mathrm{~ns}$, which is visible from the oscilloscope traces.

The scintillator signals in Fig. 7 show a time difference of roughly 150 ns between annihilation of antiprotons hitting the upstream triplet and the downstream MCP. This corresponds quite well to the calculated time of flight of $160 \mathrm{~ns}$ for $100 \mathrm{keV}$ antiprotons over the $700 \mathrm{~mm}$ separating the detectors.

The (inset) MCP images in Fig. 7 show the beam to be relatively well centered, but when decelerated by the drift tube a vertical shift of about 15 $\mathrm{mm}$ is visible (the MCP diameter is $42 \mathrm{~mm}$, inclined by 45 degrees). The beam is somewhat focused by the drift tube since it forms a long Einzel lens. Because of the possible alignment problems (mentioned at the beginning of this section) it seems plausible that the beam was not injected along the decelerator drift-tube axis, which could explain the movement of the beam under deceleration.

Because of the discharge on the drift tube mentioned earlier, we were only able to apply $-92 \mathrm{kV}$ before the maximum rated current of the supply was 


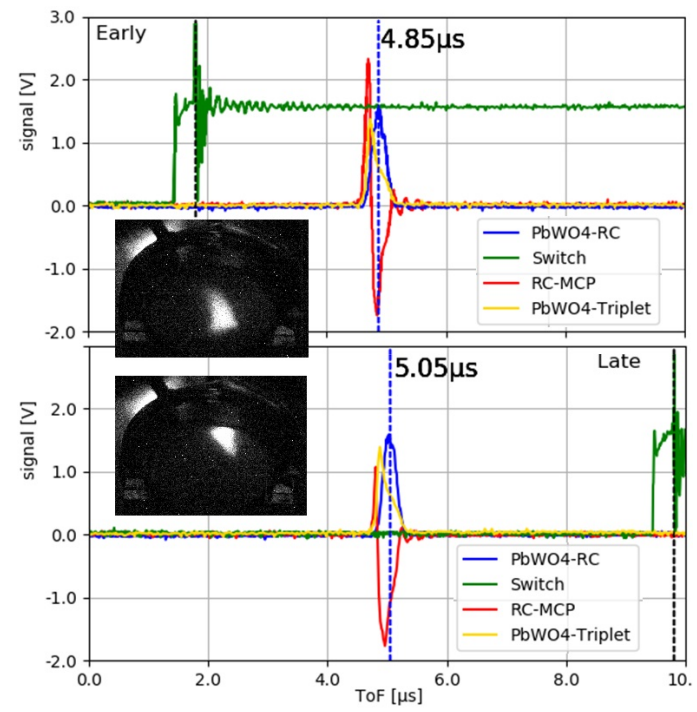

Figure 7: Static voltage operation showing detector-signal oscilloscope traces: HV-switch trigger (green - the black dotted line is the true switching time, visible from the highamplitude noise); a $\mathrm{PbWO}_{4}$ crystal located above the quadrupole triplet (yellow); a $\mathrm{PbWO}_{4}$ crystal located above the MCP (blue); the MCP signal (red). The top panel corresponds to an early trigger (no deceleration) and the bottom to a late trigger (90 keV deceleration and subsequent reacceleration to ground exiting the drift tube). The $200 \mathrm{~ns}$ ToF difference between the full-energy and slowed/re-accelerated beam annihilation signals is indicated. Insets show antiproton-beam images recorded by a CCD camera facing upwards to the inclined MCP detector.

reached. The lowest achievable beam energy was therefore only $8 \mathrm{keV}$.

In the meantime, we have learned that the drift tube insulator assembly may be insufficiently shielded, forming a so-called triple junction effect at the insulator/conductor interface [29]. This would explain not only the discharge but also the deflection of the decelerated beam, due to charging on the insulator. The "cradle" is therefore being redesigned.

Fig. 8 illustrates the deceleration of the $100 \mathrm{keV}$ ELENA beam to $8 \mathrm{keV}$ under the aforementioned conditions. On the right side of the figure, the CCD camera image of the MCP shows two distinct beam spots. We believe the upper spot corresponds to the decelerated fraction of the beam pulse and the lower spot corresponds to a "fast" (non-decelerated) fraction. This is corroborated by the static test shown in Fig. 7 where the beams are not seen at the same position because of the early and late triggering. The alignment problem mentioned earlier leads to shifts in beam position and angle which 


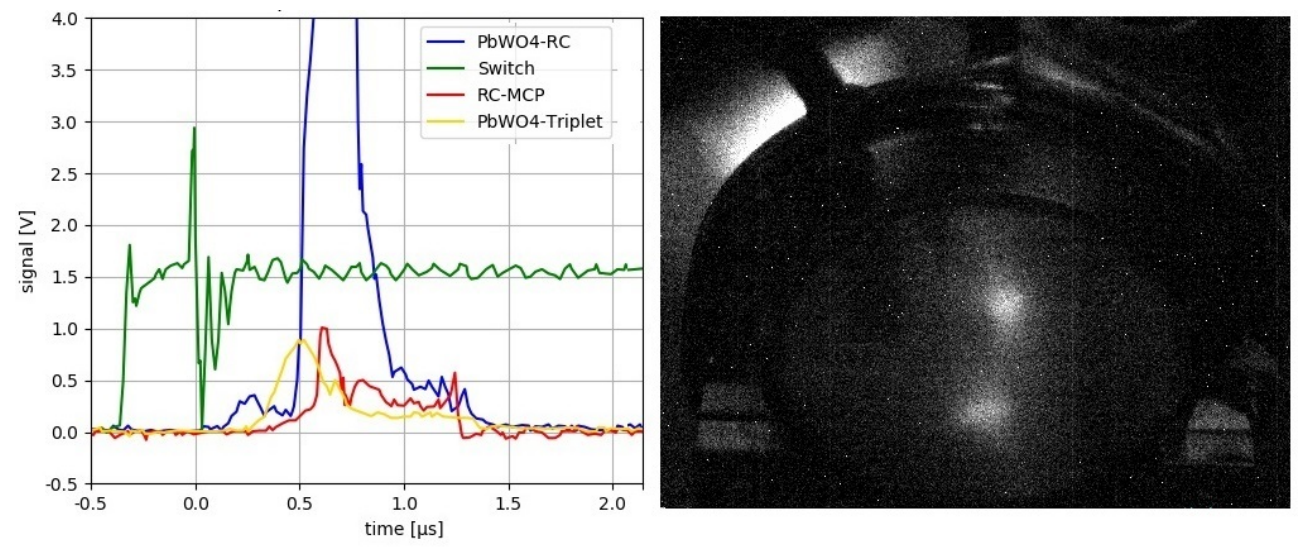

Figure 8: Demonstration of antiproton deceleration from 100 to $8 \mathrm{keV}$. As in Fig. 7 the right image was recorded by a CCD camera facing the MCP. The left panel shows recorded oscilloscope traces: Behlke-switch trigger (green); $\mathrm{PbWO}_{4}$ crystal located above the upstream lenses (yellow); $\mathrm{PbWO}_{4}$ crystal located above the MCP (blue), and MCP signal (red). The trigger was set to switch the drift-tube voltage while the pulse was inside. The MCP trace shows both the $8 \mathrm{keV}$ decelerated component (later peak) and part of the undecelerated $100 \mathrm{keV}$ beam (earlier peak). The CCD image (right) shows these two components that are spatially distinct, as seen separately in Fig. 7.

are amplified by the deceleration process. This effect has been reproduced by simulations.

But while the static test might imply that the lower spot corresponds to the decelerated antiprotons, we discovered in subsequent tests that the triple-junction effect explained by Faircloth [29] can also charge the insulator and slightly deflect the decelerated beam downward. Since the injection conditions were not the same as for the static case, it is not possible to say with absolute certainty.

The MCP signal (red trace in Fig. 8) shows two maxima with a time difference of about $650 \mathrm{~ns}$, corresponding to two groups of antiprotons: one "fast" (non-decelerated) group and one "decelerated" group. As mentioned, the reaction chamber MCP is $1170 \mathrm{~mm}$ downstream of the drift tube. The calculated time-of-flight difference for antiprotons at 100 and $8 \mathrm{keV}$ over this distance is $675 \mathrm{~ns}$, in good agreement with the detected MCP pulses shown in Fig. 8 (although the timing resolution is rather limited). There is also a continuum between the two peaks caused by antiprotons that experience the fringe field at the exit of the drift tube, convoluted with the $200 \mathrm{~ns}$ switching time. We believe this happens because the ELENA pulse length was likely 
longer than the 300 ns design value so that the entire pulse could not fit inside the drift tube before switching.

From integrating the areas under the peaks (assuming identical MCP signal response for $100 \mathrm{keV}$ and $8 \mathrm{keV}$ antiprotons) we find the decelerated fraction is about 25\% compared to the fast antiprotons. Because of the compressed commissioning schedule of ELENA, it was not possible to test different optical configurations for the decelerating lenses.

To explore the continuum effect visible in Fig. 8, ion trajectory calculations were performed to simulate the MCP signal, with the results shown in Fig. 9. As during the experimental tests, $-92 \mathrm{kV}$ was applied to the drift

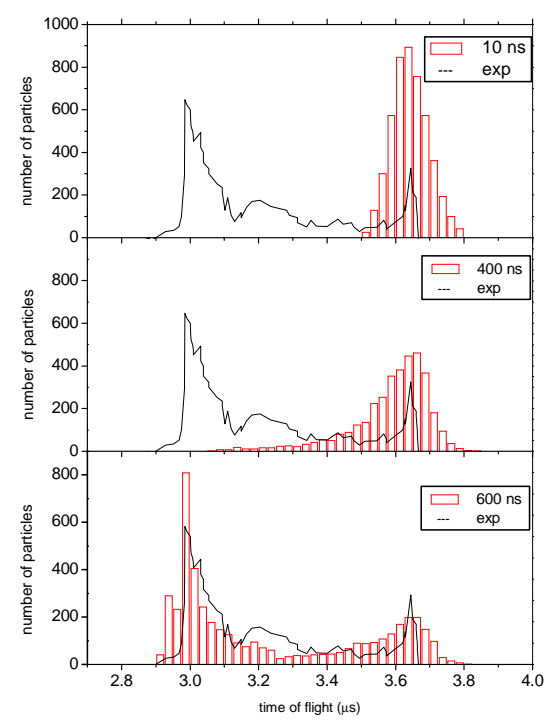

Figure 9: Simulated time-of-flight histograms (red columns) of antiprotons decelerated from $100 \mathrm{keV}$ to $8 \mathrm{keV}$ with different injected pulse durations. Top panel shows a beam pulse of only $10 \mathrm{~ns}$ for reference. The middle panel shows a $400 \mathrm{~ns}$ pulse that results in a decelerated pulse of $497 \mathrm{~mm}$ in length, which clearly does not fit into the drift tube. The bottom panel shows a $600 \mathrm{~ns}$ pulse that produces decelerated and re-accelerated beam components. The recorded MCP pulse is also shown (solid back line).

tube with the other decelerating lenses at ground. The three panels show different input pulse durations of 10, 400 and 600 ns (the design value is 300 ns [15]). The switching pulses were modeled using an exponential decay, with 
the measured time constant of 200 ns. The switch is always triggered while the beam is centered in the drift tube. For the hypothetical case of $10 \mathrm{~ns}$ (top panel), a single decelerated pulse is seen, as expected. The increased width of the peak is due to the large energy spread quoted in the ELENA design [15]. When the beam occupies the areas near the drift-tube edges (middle panel), the antiprotons experience varying amounts of re-acceleration and "leak out" of the main peak to earlier arrival times. With the pulse protruding even farther out of both ends of the drift tube (bottom panel), the continuum seen in the measurements (Fig. 8, left panel) appears. The recorded MCP signal (slightly shifted in time and scaled in amplitude) is also included in the figure for comparison.

The simulations showing the $100 \mathrm{keV}$ and $8 \mathrm{keV}$ antiproton-deceleration peaks agree with the measured 650 ns time-of-flight difference to within about $25 \mathrm{~ns}$, or better than $4 \%$. The ELENA pulse length was not monitored during the tests. A CERN internal note concerning low-level RF tests during the ELENA commissioning reports a typical pulse of 600 ns that was improved at one stage to $200 \mathrm{~ns}$ (see Fig. 34 in [30]). The 600 ns pulse length qualitatively matches our measurements.

Ref. [15] quotes an estimated momentum spread of $0.25 \%$, which would correspond to a very large $500 \mathrm{eV}$ energy spread. A more optimistic energy spread of only $100 \mathrm{eV}$ was used in the simulations. The simulated peaks have larger widths compared to the measurement, which may indicate that the projected ELENA energy spread of $0.5 \%$ is too conservative.

In a final test, the decelerated antiprotons were transported $2 \mathrm{~m}$ downstream of the reaction chamber, through a beam switchyard designed to separate unreacted antiprotons from neutral antihydrogen and the antihydrogen ions (see chapter 6 of [23]). An antiproton signal was detected with an MCP mounted on the straight section and could be moved laterally by applying the switchyard-electrode voltages (see sections 7.5 and 8.5 of [31]). Again, due to experimental restrictions, we could not obtain quantitative information.

\section{Summary}

We have presented a new scheme using electrostatic optics and fast highvoltage switching for decelerating antiproton bunches down to keV energies for precision experiments such as GBAR at the CERN AD facility. Such a scheme avoids losses associated with passing the beam through thin foils, and 
should offer increased efficiency. A detailed technical description of the decelerator design and realization has been given. Prior to CERN's second Long Shutdown (LS2) we successfully commissioned the first $100 \mathrm{keV}$ pulsed drift tube and electrostatic-lens system built to decelerate the pulsed antiproton beam from the ELENA storage ring of CERN's AD "antimatter factory". Because the tests were performed during the very short commissioning of ELENA, only limited results could be obtained. Our results give clear evidence of antiproton pulses decelerated to $8 \mathrm{keV}$. Since the ELENA pulses were longer than the design value of $300 \mathrm{~ns}$, only a fraction of the antiprotons were decelerated. This situation will be improved when CERN restarts in mid-2021.

\section{Acknowledgements}

We thank C. Bachelet, J. Bourçois, J. Bui, S. Cabaret, P. Dupré, P. Duarte, S. Hervé, D. Le Du, H. Lefort, A. Limongi, V. Manea, S. Martineau, K. Nguyen, S. Pitrel and H. Ramirijaona from the CSNSM, C. Doré and J.-M. Isac from the LKB, J.-L. Babigeon from the LAL, D. Desforges, Ph. Hardy, S. Javello, D. Pierrepont and C. Vuillemin from the CEA and F. Butin from CERN for their various contributions. We also thank the ELENA team for their help and cooperation during the tests.

The decelerator project was supported by France's IN2P3, by the Agence National de Recherche under project no. ANR-14-CE33-0008 (ANTION), and by the Laboratoire d'Excellence P2IO (ANR-10-LABX-0038) in the framework Investissements d'Avenir (ANR-11-IDEX-0003-01).

\section{References}

[1] P. Pérez, et al., The gbar antimatter gravity experiment, Hyperfine interactions 233 (2015) 21-27.

[2] C. Amole, et al., Description and first application of a new technique to measure the gravitational mass of antihydrogen, Nature Communications 4 (2013) 1785.

[3] S. Aghion, et al., A moiré deflectometer for antimatter, Nature Communications 5 (2014) 4538. 
[4] C. Monroe, D. Meekhof, B. King, S. R. Jefferts, W. M. Itano, D. J. Wineland, P. Gould, Resolved-sideband raman cooling of a bound atom to the 3d zero-point energy, Physical Review Letters 75 (1995) 4011.

[5] L. Hilico, J.-P. Karr, A. Douillet, P. Indelicato, S. Wolf, F. Schmidt Kaler, Preparing single ultra-cold antihydrogen atoms for free-fall in gbar, in: International Journal of Modern Physics: Conference Series, volume 30, World Scientific, p. 1460269.

[6] N. Sillitoe, J.-P. Karr, J. Heinrich, T. Louvradoux, A. Douillet, L. Hilico, Sympathetic cooling simulations with a variable time step, in: Proceedings of the 12th International Conference on Low Energy Antiproton Physics (LEAP2016), p. 011014.

[7] L. Liszkay, M.-F. Barthe, C. Corbel, P. Crivelli, P. Desgardin, M. Etienne, T. Ohdaira, P. Pérez, R. Suzuki, V. Valtchev, A. Walcarius, Orthopositronium annihilation and emission in mesostructured thin silica and silicalite-1 films, Applied Surface Science 255 (2008) 187.

[8] L. Liszkay, with the GBAR Collaboration, Positron production using a 9 mev electron linac for the GBAR experiment, Nucl. Instrum. Meth. A 985 (2021) 164657.

[9] S. Niang, et al., Accumulation of positrons from a linac based source., Acta Physica Polonica, A. 137 (2020) 164.

[10] J. Humberston, M. Charlton, F. Jacobsen, B. Deutch, On antihydrogen formation in collisions of antiprotons with positronium, J. Phys. B: At. Mol. Phys. 20 (1987) L25.

[11] J. P. Merrison, H. Bluhme, J. Chevallier, B. I. Deutch, P. Hvelplund, L. V. Jorgensen, H. Knudsen, M. R. Poulsen, M. Charlton, Hydrogen formation by proton impact on positronium, Phys. Rev. Lett. 78 (1997) 2728 .

[12] P. Comini, P.-A. Hervieux, $\overline{\mathrm{H}}+$ ion production from collisions between antiprotons and excited positronium: cross sections calculations in the framework of the GBAR experiment, New Journal of Physics 15 (2013) 095022 . 
[13] A. S. Kadyrov, C. M. Rawlins, A. T. Stelbovics, I. Bray, M. Charlton, Antihydrogen formation via antiproton scattering with excited positronium, Phys. Rev. Lett. 114 (2015) 183201.

[14] T. Yamashita, Y. Kino, E. Hiyama, K. Piszczatowski, S. Jonsell, P. Froelich, Towards prediction of the rates of antihydrogen positive ion production in collision of antihydrogen with excited positronium, Journal of Physics: Conference Series 1412 (2020) 052012.

[15] W. Bartmann, P. Belochitskii, H. Breuker, F. Butin, C. Carli, T. Eriksson, W. Oelert, R. Ostojic, S. Pasinelli, G. Tranquille, The ELENA facility, Phil. Trans. R. Soc. A 376 (2017) 20170266.

[16] G. Gabrielse, X. Fei, K. Helmerson, S. L. Rolston, R. Tjoelker, T. A. Trainor, H. Kalinowsky, J. Haas, W. Kells, First capture of antiprotons in a penning trap: A kiloelectronvolt source, Phys. Rev. Lett. 57 (1986) $2504-2507$.

[17] G. Gabrielse, N. Bowden, P. Oxley, A. Speck, C. Storry, J. Tan, M. Wessels, D. Grzonka, W. Oelert, G. Schepers, T. Sefzick, J. Walz, H. Pittner, T. Haensch, E. Hessels, Stacking of cold antiprotons, Physics Letters B 548 (2002) $140-145$.

[18] M. Hori, The asacusa experiment at cern's antiproton decelerator, Nucl. Phys. A 692 (2001) $119-128$.

[19] N. Kuroda, H. A. Torii, K. Y. Franzen, Z. Wang, S. Yoneda, M. Inoue, M. Hori, B. Juhász, D. Horváth, H. Higaki, A. Mohri, J. Eades, K. Komaki, Y. Yamazaki, Confinement of a large number of antiprotons and production of an ultraslow antiproton beam, Phys. Rev. Lett. 94 (2005) 023401.

[20] N. Kuroda, H. A. Torii, Y. Nagata, M. Shibata, Y. Enomoto, H. Imao, Y. Kanai, M. Hori, H. Saitoh, H. Higaki, A. Mohri, K. Fujii, C. H. Kim, Y. Matsuda, K. Michishio, Y. Nagashima, M. Ohtsuka, K. Tanaka, Y. Yamazaki, Development of a monoenergetic ultraslow antiproton beam source for high-precision investigation, Phys. Rev. ST Accel. Beams 15 (2012) 024702.

[21] D. Lunney, P. Dupré, P. Grandemange, V. Manea, T. Mortensen, S. Cabaret, S. Pitrel, P. Comini, P. Debu, L. Liszkay, P. Lotrus, P. Pérez, 
J.-M. Rey, J.-M. Reymond, N. Ruiz, Y. Sacquin, B. Vallage, D. BrookRoberge, P. Hardy, Beam preparation for studying the gravitational behavior of antimatter at rest (GBAR), Hyp. Interact. 229 (2014) 1.

[22] A. Husson, D. Lunney, An antiproton deceleration device for the GBAR experiment at CERN, arXiv:1909.07493 (2016).

[23] A. Husson, Deceleration of antiprotons from cern's elena synchrotron and transport of antimatter beams through the gbar experiment, Doctoral Thesis, Université Paris-Saclay (2018) http://cds.cern.ch/record/2712543.

[24] F. Herfurth, J. Dilling, A. Kellerbauer, G. Bollen, S. Henry, H.-J. Kluge, E. Lamour, D. Lunney, R. B. Moore, S. Scheidenberger, S. Schwarz, G. Sikler, J. Szerypo, A linear radiofrequency ion trap for accumulation, bunching, and emittance improvement of radioactive ion beams, Nucl. Instrum. Meth. A 469 (2001) 254.

[25] A. M. Ghalambor Dezfuli, Injection, cooling and extraction of ions from a very large paul trap, Ph.D. thesis, McGill University (1996).

[26] S. Coeck, B. Delaur, M. Herbane, M. Beck, V. Golovko, S. Kopecky, V. Kozlov, I. Kraev, A. Lindroth, T. Phalet, D. Beck, P. Delahaye, A. Herlert, F. Wenander, N. Severijns, A pulsed drift cavity to capture $30 \mathrm{kev}$ ion bunches at ground potential, Nuclear Instruments and Methods in Physics Research Section A: Accelerators, Spectrometers, Detectors and Associated Equipment 572 (2007) 585 - 595.

[27] J. Grund, M. Asai, K. Blaum, M. Block, S. Chenmarev, C. Dllmann, K. Eberhardt, S. Lohse, Y. Nagame, S. Nagy, P. Naubereit, J. van de Laar, F. Schneider, T. Sato, N. Sato, D. Simonovski, K. Tsukada, K. Wendt, First online operation of triga-trap, Nuclear Instruments and Methods in Physics Research Section A: Accelerators, Spectrometers, Detectors and Associated Equipment 972 (2020) 164013.

[28] C. Smorra, High-precision Q-value and mass measurements for neutrino physics with TRIGA-TRAP and commissioning of an on-line ion source for triga-spec, Ph.D. thesis, University of Heidelberg (2012). 
[29] D. C. Faircloth, Technological Aspects: High Voltage, in CAS - CERN Accelerator School: Ion Sources 10.5170/CERN-2013-007.381 (2013) 381-419, https://cds.cern.ch/record/1693330.

[30] M. E. Angoletta, M. Jaussi, J. Molendijk, New llrf capabilities and beam results for the second year of elenas commissioning, CERN-ACCNOTE-2019-0050 (2019) https://cds.cern.ch/record/2703432.

[31] B. Latacz, Study of the antihydrogen atom and ion production via charge exchange reaction on positronium, Doctoral Thesis, Université Paris-Saclay (2019) https://tel.archives-ouvertes.fr/tel-02417434/document. 


\section{The GBAR Collaboration}

A. Husson ${ }^{\mathrm{a}, 1}$, B. H. Kim ${ }^{\mathrm{b}}$, A. Welker ${ }^{\mathrm{c}}$, M. Charlton $^{\mathrm{d}}$, J. J. Choi ${ }^{\mathrm{b}}$, M. Chung ${ }^{e}$, P. Cladéf ${ }^{\prime}$ P. Comini ${ }^{h}$, P-P. Crépin ${ }^{f}$, P. Crivelli ${ }^{i}$, O. Dalkarov ${ }^{j}$, P. Debu ${ }^{h}$, L. Dodd ${ }^{d}$, A. Douillet ${ }^{f, g}$, S. Guellati-Khélifa ${ }^{f}$, N. Garroum ${ }^{f}$, P-A. Hervieux ${ }^{k}$, L. Hilico ${ }^{f, g}$, P. Indelicato ${ }^{f}$, G. Janka ${ }^{i}$, S. Jonsell ${ }^{l}$, J-P. Karr ${ }^{f, g}$, E-S. Kim ${ }^{\mathrm{m}}$, S. K. Kim ${ }^{\mathrm{b}}$, Y. Ko ${ }^{\mathrm{n}}$, T. Kosinski ${ }^{\mathrm{O}}$, N. Kuroda ${ }^{\mathrm{p}}$, B. Latacz ${ }^{\mathrm{h}, 2}$, H. Lee ${ }^{\mathrm{b}}$, J. Lee ${ }^{\mathrm{n}}$, A. M. M. Leite ${ }^{\mathrm{h}, 3}$, K. Lévêque $^{\mathrm{k}}$, E. Lim $^{\mathrm{m}}$, L. Liszkay ${ }^{h}$, P. Lotrus ${ }^{h}$, T. Louvradoux ${ }^{f}$, D. Lunney ${ }^{*, a}$, G. Manfredik, B. Mansouliéh ${ }^{\text {, M. Matusiak }}$, G. Mornacchic ${ }^{\mathrm{c}}$ V. V. Nesvizhevsky ${ }^{\mathrm{q}}$, F. Nez ${ }^{\mathrm{f}}$,

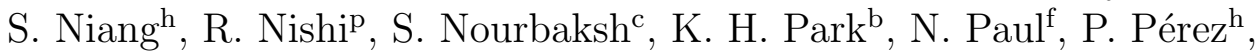
S. Procureur ${ }^{h}$, B. Radics ${ }^{i}$, C. Regenfus ${ }^{i}$, J-M. Reymond ${ }^{h}$, S. Reynaud ${ }^{f}$, J-Y. Rousséh ${ }^{\text {, O. Rousselle }}$, A. Rubbiai, J. Rzadkiewicz ${ }^{\mathrm{o}}$, Y. Sacquin ${ }^{\mathrm{h}}$, F. Schmidt-Kaler ${ }^{r}$, M. Staszczak ${ }^{\mathrm{o}}$, B. Tuchming ${ }^{\mathrm{h}}$, B. Vallage ${ }^{\mathrm{h}}$, A. Voronin ${ }^{\mathrm{j}}$,

D. P. van der Werf ${ }^{\mathrm{d}}$, S. Wolf ${ }^{\mathrm{r}}$, D. Won ${ }^{\mathrm{b}}, \mathrm{S}$. Wronka ${ }^{\mathrm{o}}$, Y. Yamazaki ${ }^{\mathrm{s}}$, K-H. Yoo

${ }^{a}$ IJCLab, IN2P3/CNRS-Université Paris-Saclay, 91405 Orsay, France

${ }^{b}$ Department of Physics and Astronomy, Seoul National University, 599 Gwanak-Ro, Gwanak-gu, Seoul 08826, Korea

${ }^{c}$ CERN, 1211 Geneva 23, Switzerland

${ }^{d}$ Department of Physics, College of Science, Swansea University, Swansea SA2 8PP, United Kingdom

${ }^{e}$ Department of Physics, Ulsan National Institute of Science and Technology (UNIST), 50, UNIST-gil, Ulsan 44919, Republic of Korea

${ }^{f}$ Laboratoire Kastler Brossel, Sorbonne Université, CNRS, ENS-PSL Research University, Collège de France, Case 74, 4, place Jussieu, F-75005 Paris, France ${ }^{g}$ Université d'Évry-Val d'Essonne, Université Paris-Saclay, Boulevard François Mitterrand, F-91000 Évry, France

${ }^{h} I R F U, C E A$, Université Paris-Saclay, F-91191 Gif-sur-Yvette Cedex, France

${ }^{i}$ Institute for Particle Physics and Astrophysics, ETH Zürich, CH-8093 Zürich, Switzerland

\footnotetext{
${ }^{*}$ Corresponding author.

Email address: david.lunney@ijclab.in2p3.fr (D. Lunney)

${ }^{1}$ present address: CENBG, 19 Chemin du Solarium, CS 10120, F-33175 Grandignan, France

${ }^{2}$ present address: RIKEN, Ulmer Fundamental Symmetries Laboratory, Wako, Saitama 351-0198, Japan

${ }^{3}$ present address: Institut Curie, PSL Research University, Radiation Oncology Department, Proton Therapy Centre, Centre Universitaire, 91898, Orsay, France
} 
${ }^{j}$ P. N. Lebedev Physical Institute, 53 Leninsky Prospect, 117991 Moscow, Russia

${ }^{k}$ Université de Strasbourg, CNRS, Institut de Physique et Chimie des Matériaux de Strasbourg, UMR 7504, F-67000 Strasbourg, France

${ }^{l}$ Department of Physics, Stockholm University, SE-10691 Stockholm, Sweden

${ }^{m}$ Department of Accelerator Science, Korea University Sejong Campus, Sejong-ro 2511, 0019 Sejong, Republic of Korea

${ }^{n}$ Center for Underground Physics, Institute for Basic Science, 70 Yuseong-daero 1689-gil, Yuseong-gu, Daejeon 34047, Korea

${ }^{\circ}$ National Centre for Nuclear Research (NCBJ), ul. Andrzeja So?tana 7, 05-400 Otwock, Swierk, Poland

${ }^{p}$ Institute of Physics, University of Tokyo, 3-8-1 Komaba, Meguro, Tokyo 153-8902, Japan

${ }^{q}$ Institut Max von Laue - Paul Langevin (ILL), 71 avenue des Martyrs, F-38042 Grenoble, France

${ }^{r}$ QUANTUM, Institut für Physik, Johannes Gutenberg Universität, D-55128 Mainz, Germany

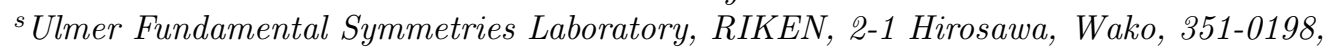
Saitama, Japan 